\section{The Royal College of Ophthalmologists Guidelines on retinal vein occlusions: executive summary}

\author{
This article has been corrected since Advance Online \\ Publication and a corrigendum is also printed in this issue
}

Retinal vein occlusion (RVO) is the second most common retinal vascular disease after diabetic retinopathy. Central retinal vein occlusion (CRVO) results from thrombosis of the central retinal vein when it passes through the lamina cribrosa. ${ }^{1,2}$ It is classically characterised by disc oedema, increased dilatation, and tortuosity of all retinal veins, widespread deep and superficial haemorrhages, cotton wool spots, retinal oedema, and capillary nonperfusion in all four quadrants of the retina. In less severe forms, the disc oedema may be absent. A previous CRVO may show evidence of optic disc and retinal collaterals, a telangiectatic capillary bed, and persistent venous dilation and tortuosity, peri-venous sheathing, arteriolar narrowing, and macular abnormalities (chronic macular oedema and retinal pigment epithelial changes). Branch retinal vein occlusion (BRVO) is caused by venous thrombosis at an arteriovenous crossing where an artery and vein share a common vascular sheath. ${ }^{3,4}$ It has similar features to CRVO except that the findings are confined to that portion of the fundus drained by the affected vein. Hemiretinal vein occlusion (HRVO) affects either the superior or inferior retinal hemisphere, and the retinal haemorrhages are nearly equal in two altitudinal quadrants (the nasal and temporal aspects) of the involved hemisphere.

The two main complications of RVO are macular oedema (MO), and retinal ischaemia leading to iris and retinal neovascularisation. Thrombosis of the retinal veins cause an increase in retinal capillary pressure resulting in increased capillary permeability and leakage of fluid and blood into the retina. Coexistent retinal ischaemia may exacerbate this process by the production of vascular endothelial growth factor (VEGF), which in turn promotes retinal capillary permeability and leakage into the extracellular space resulting in further development of $\mathrm{MO} . \mathrm{MO}$ is the most common cause of visual impairment in $\mathrm{RVO}$, followed by foveal ischaemia. Varying degrees of retinal ischaemia due to nonperfusion of retinal capillaries may occur and principally depends on the degree of retinal vein thrombosis. These changes result in increased production of VEGF and other cytokines, which promote new vessel formation principally, but not exclusively, involving the iris and angle in CRVO and the retina in BRVO. These complications may lead to neovascular glaucoma, vitreous haemorrhage, and tractional retinal detachment (depending on where the neovascularisation occurs) with severe visual impairment.

Both CRVO and BRVO can be broadly classified into ischaemic and nonischaemic types based on the area of capillary nonperfusion. The Central Retinal Vein Occlusion Study (CVOS) defined ischaemic CRVO as fluorescein angiographic evidence of $>10$ disc areas of capillary nonperfusion on seven-field fundus fluorescein angiography. 5,6 However, this definition may require revision to be appropriate for the more recently adopted wide-angle imaging. It is important that a clear distinction is made between foveal ischaemia and an ischaemic RVO (ie, global retinal ischaemia).

Ischaemic CRVO is associated with one or more of the following characteristics: ${ }^{5,6}$ poor visual acuity of $<6 / 60$; relative afferent pupillary defect; presence of multiple dark deep intraretinal haemorrhages, presence of multiple cotton wool spots, degree of retinal vein dilatation and tortuosity, and fluorescein angiography showing $>10$ disc areas of retinal capillary nonperfusion on seven-field fluorescein angiography; other features include electrodiagnostic tests (particularly electroretinogramERG) which show reduced b-wave amplitude, reduced $\mathrm{b}$ : a ratio and prolonged $\mathrm{b}$-wave implicit time on the electroretinogram. ${ }^{7-11}$ There is no evidence as to which combination of the above characteristics best defines ischaemic CRVO. It is important to note that up to $30 \%$ of eyes with initially nonischaemic CRVO may convert to ischaemic subtype. ${ }^{11-14}$ This is usually heralded by further rapid visual deterioration and requires additional assessment.

\section{Natural history \\ Central retinal vein occlusion}

Although some patients with CRVO can experience an improvement in $\mathrm{MO}$ and visual acuity, visual acuity generally decreases over time. Only $20 \%$ of patients who present with an initial visual acuity of 35-65 ETDRS letters (Snellen equivalent 6/60 to 6/18) are likely to improve spontaneously.

Nonischaemic CRVO may resolve completely without any complications. Follow-up of such cases for at least 2 years is usually recommended; however, the development of disc collaterals and the resolution of $\mathrm{MO}$ for at least 6 months may indicate adequate reperfusion (although not fool proof), and should allow the discharge of the patient from clinical supervision. Approximately, $30 \%$ may convert to an ischaemic CRVO over 3 years owing to an increase in area of nonperfusion. More than $90 \%$ of patients with ischaemic CRVO have a final visual acuity of $6 / 60$ or worse. ${ }^{5,6}$ CRVO in younger patients $(<50$ years of age) has been thought to have a more benign outcome in a greater proportion of patients, with spontaneous regression of the venous occlusive event being more common. However, at least $20 \%$ of patients develop poor visual outcome with severe neovascular complications. The causation of RVO in the younger person is still unclear in most cases. A role for dehydration in such cases has been suggested but remains unproven.

\section{Branch retinal vein occlusion}

On the basis of the Branch Retinal Vein Occlusion study (BVOS) ${ }^{15}$ the prognosis of BRVO is better than CRVO with approximately $50-60 \%$ of untreated BRVO cases retaining a visual acuity $\geq 6 / 12$ after 1 year.

Therefore, when a patient presents with recent onset mild visual impairment due to MO secondary to BRVO, it may be reasonable to observe the progress of the condition over the first 3 months of follow-up. Approximately, $20 \%$ of untreated MO due to BRVO experience significant deterioration of visual acuity over time. ${ }^{15}$ The majority of RVO cases present as a unilateral condition. However, 5-6\% present with evidence of 
bilateral BRVO and $10 \%$ of the BRVO patients will have fellow eye involvement over time. Similarly, $~ 10 \%$ of CRVO patients present with bilateral involvement at baseline and 5\% may have fellow eye involvement over a 1-year period. ${ }^{16}$

\section{Hemiretinal vein occlusion}

The risk of rubeosis in ischaemic HRVO is greater than that of BRVO, but less than that of CRVO. ${ }^{12}$ The risk of disc neovascularisation appears greater for hemispheric vein occlusion than either ischaemic CRVO or BRVO. The management of hemispheric vein occlusion is similar to that described for branch retinal vein occlusion.

\section{Risk factors}

RVO is due to thrombosis of retinal veins (central, hemi, or branch). ${ }^{1,2}$ Atherosclerosis of the adjacent central retinal artery possibly compresses the central retinal vein at the lamina cribrosa leading to consequent thrombosis in the venous lumen. The risk factors of RVO include hypertension, diabetes, hyperlipidemia, hyperhomocysteinaemia, blood dyscrasias, systemic inflammatory diseases, glaucoma, and shorter axial length. Rarely, retrobulbar external compression from thyroid eye disease, orbital tumour, or retrobulbar haemorrhage may be a cause.

\section{Medical investigations of RVO}

In general, the aims of investigations in a medical condition are to allow potential treatment of causative factors that might lead to improvement of the condition, prevent progression or prevent recurrence in the same eye or in the other eye, and to reduce the risk to overall health. Apart from the rarely associated hyperviscosity conditions, there is little evidence that the natural history of a RVO is influenced, or a further RVO prevented by a battery of investigations. The main benefit of medical tests in RVO is to improve health by treating the commonly associated risk factors of atherosclerosis, hypertension, diabetes, and lipid abnormalities.

\section{Summary of recommended medical investigations in the eye clinic}

Checking the BP, serum glucose, FBC and ESR will detect associations with RVO that require urgent action such as severe hypertension, uncontrolled diabetes with end-organ damage, or rarely, blood conditions such as leukaemia. A raised ESR may point to an inflammatory condition or a blood disorder such as myeloma.

Further assessment of potential associated conditions, including further medical tests, are probably best performed by the patient's physician who can then organise further management and supportive measures such as smoking cessation.

The history, ocular examination and initial test results may direct further investigations. For example, raised plasma viscosity or raised white cells could point to Waldenstrom's macroglobulinaemia, such that questions about symptoms such as night sweats, a physical examination for lymphadenopathy, and immunoglobulin electrophoresis may be indicated.
Bilateral presentation or any sign of a vascular disturbance in the other eye, such as a few dot haemorrhages should increase suspicion of an underlying systemic condition.

The British Society of Haematology does not recommend routine thrombophilia testing for RVO. Testing for acquired thrombophilia in an isolated RVO is also not recommended. ${ }^{17}$

It is recommended that oestrogen-containing hormone replacement therapy and oral contraceptives not be commenced in those women with a history of RVO. However, the continued use in a patient who develops RVO does not appear to be associated with a higher rate of recurrence. The decision about whether to continue these oestrogen-containing therapies in a woman with RVO should be made on a case by case basis.

\section{Current treatment options for macular oedema due to RVO}

\section{Laser photocoagulation}

The CVOS study failed to indicate benefit from grid laser treatment, although a trend in favour of treatment was observed in younger patients. ${ }^{18}$ There is also no evidence to suggest any benefit from a combination of macular grid laser and intravitreal anti-VEGF or steroids for MO secondary to CRVO at the present. The BVOS study showed that, after 3 years of follow-up and based on available data on 43 participants, 28 (63\%) of laser-treated eyes had improved $\geq 2$ lines of vision, compared with 13 $(37 \%)$ out of 35 untreated eyes that remained in the study for 36 months. ${ }^{19}$

\section{Intravitreal steroids}

The rationale for the use of steroids as treatment for $\mathrm{MO}$ is that corticosteroids reduce retinal capillary permeability and inhibit the expression of the VEGF gene and the metabolic pathway of VEGF.

On the basis of the GENEVA study programme, Dexamethasone implant (Ozurdex, Allergan) has received FDA and EU approval for the $0.7-\mathrm{mg}$ preparation, and is licensed in the UK for the treatment of adult patients with $\mathrm{MO}$ following CRVO and BRVO. ${ }^{20}$ NICE TA 229 has recommended the use of Ozurdex in the treatment of MO secondary to CRVO and BRVO.

\section{Anti-VEGF agents}

These agents are now a popular choice for treatment of $\mathrm{MO}$ due to RVO based on the fact that VEGF-A is a key cytokine that mediates vascular leakage and causes $\mathrm{MO}$ in RVO. Intraocular VEGF levels are significantly high in CRVO compared with controls. The pan-VEGF-A blocker, ranibizumab (Lucentis, Novartis) is EMA approved and recommended by NICE (NICE TA283, May 2013) for the treatment of visual impairment due to MO secondary to CRVO and BRVO. ${ }^{21-23}$ Aflibercept (Eylea, Bayer), a recombinant fusion protein that blocks VEGF-A, B and Placental growth factor (PIGF) is also EMA approved for MO owing to CRVO and BRVO, ${ }^{24-26}$ and is recommended by NICE (NICE TA 305) for CRVO. The NICE appraisal of aflibercept for BRVO is awaited. 
Treatment algorithm for CRVO

A. Treatment of risk factors (to be managed by patient's physician).

B. Ophthalmic management.

\section{Nonischaemic CRVO}

Baseline. Visual acuity measurement, colour fundus photographs, and fluorescein angiography, OCT, intraocular pressure (IOP), gonioscopy (if ischaemic CRVO is suspected) are recommended.

If no iris or angle NV is noted and there is OCT evidence of MO:

(a) If visual acuity is $6 / 96$ or better, then commence on either intravitreal anti-VEGF therapy, or Ozurdex implant.

(b) If visual acuity is $<6 / 96$, then the potential for significant improvement in visual acuity is minimal and the risk of ocular neovascularisation is high.

However, eyes with VA $<6 / 96$ may be offered treatment as some of these eyes may respond. The patients should be watched for NVI/NVA.

(c) If visual acuity is better than $6 / 12$, then it is reasonable to observe the patient for spontaneous resolution as per the judgment of the treating ophthalmologist.

Choice of agent. Ranibizumab and aflibercept are the two anti-VEGF agents recommended by NICE for MO owing to CRVO. Dexamathasone implant (Ozurdex, Allergan) is also recommended by NICE for this condition. There is no visual acuity or central macular thickness restriction in the commencement of treatment with any of these agents.

Although any of these drugs may be used as first line for this condition, anti-VEGF is preferred in eyes with a previous history of glaucoma and younger patients who are phakic. Ozurdex may be a better choice in patients with recent cardiovascular events and in those who do not favour monthly injections.

Retreatment. At each follow-up visit, visual acuity, OCT macular thickness and IOP should be assessed and the presence of NVI/NVA assessed.

If ranibizumab is the first line of treatment, then monthly intravitreal injections are continued until maximum visual acuity is achieved, which is defined as stable visual acuity for three consecutive monthly assessments while on ranibizumab therapy. If no improvement in visual acuity over the course of the first three injections is observed, then cessation of treatment may be considered and is recommended after six injections. Patients who achieve visual acuity stability should be monitored monthly and treatment with ranibizumab is resumed when monitoring indicates loss of visual acuity due to MO secondary to CRVO. Monthly injections should then be administered again until stable visual acuity is reached for three consecutive monthly assessments (implying a minimum of two injections). The interval between two doses should not be shorter than one month.

If aflibercept is chosen as the first line treatment, then it is given monthly until maximum visual acuity is achieved, which is defined as stable visual acuity for three consecutive monthly assessments while on aflibercept therapy. If no improvement in visual acuity over the course of the first three injections is observed, then cessation of treatment may be considered and is recommended after six injections. Monthly treatment should continue until visual and anatomical outcomes are stable for three monthly assessments. Thereafter, the need for continued treatment should be reconsidered. The summary of product characteristics states that monitoring is recommended at the injection visits and that the monitoring schedule should be determined by the doctor responsible for the patient's care based on the response of the condition to treatment.

If Ozurdex is the first line of treatment, then retreatment may be required at four to six monthly intervals until visual stability is obtained. The occasional patient may require treatment at 3 months. However, more frequent and repeated treatments with Ozurdex increase the risk of adverse events and these should be discussed with the patient. Patients should be monitored for raised IOP and formation or progression of cataract.

Stopping treatment. Stopping ranibizumab and aflibercept therapy should be considered if after three consecutive monthly treatments, visual acuity has not improved by at least five letters and CMT has not reduced from baseline. However, reduction in retinal oedema without VA improvement or deterioration (ie, stable VA) may be accepted as a favourable, but suboptimal outcome. Stopping ranibizumab and aflibercept therapy is definitely recommended if after six consecutive monthly treatments, visual acuity has not improved by at least five letters and/or CMT has not reduced from baseline. Ozurdex should be used with caution in eyes with raised IOP.

Switching agents. If an anti-VEGF agent is stopped owing to lack of efficacy, there is no randomised controlled trials that provide evidence that switching to another anti-VEGF agent may be effective. However, given our experience with switching anti-VEGF agents in neovascular age related macular degeneration, it may be worthwhile switching to another anti-VEGF agent and further monthly injections for 3 months may be given to assess the efficacy of the switch.

There is a good rationale to switch from Ozurdex to an anti-VEGF agent and vice versa as the different mode of actions of these agents may aid in resolution of MO. However, the long-term outcomes of sequential or combination treatment of anti-VEGF agents and steroids remain unclear.

\section{Ischaemic CRVO}

If iris or angle neovascularisation occurs and the anterior chamber angle is open. Urgent PRP is recommended and with review at 2 weeks initially and then less frequently as regress of NV occurs. PRP plus intravitreal 
bevacizumab (off-licence) can be repeated if NVI/NVA persist.

If iris or angle $N V$ are present with a closed angle and raised IOP. Urgent PRP is recommended with cyclodiode-laser therapy/tube-shunt surgery. The latter is preferable if the angle closure is established. If the IOP is normal or normalises with the above therapy, intravitreal

bevacizumab can be considered. If the IOP is significantly elevated, then it should be managed as above with topical and medical management in addition. Caution is advised if bevacizumab or any anti-VEGF agent is considered in the presence of raised IOP as this can be exacerbated in the short term. If vitreous haemorrhage precludes a view of the fundus, then transcleral diode therapy and retinal cryotherapy can be used. An early specialist glaucoma opinion should be sought.

If an ischaemic CRVO is present without NVI/NVG and limited follow-up is likely and especially but not necessarily only if the FFA shows $>30$ DA nonperfusion, then prophylactic PRP should be considered. MO in eyes with ischaemic CRVO is treated in the same way as those with nonischaemic CRVO. However, the guarded prognosis should be explained to the patient.

\section{Treatment algorithm for BRVO}

(a) Treatment of risk factors by patient's physician.

(b) Ophthalmic management of BRVO.

\section{Nonischaemic BRVO \\ Baseline}

1. If VA better than $6 / 12$, then it is reasonable to regularly observe progress for 3 months.

2. If $\mathrm{VA}$ is $6 / 12$ or worse with $\mathrm{MO}$ and haemorrhages are not masking fovea:

(a) FFA is recommended to assess foveal integrity.

(b) If no macular ischaemia is identified, regularly observe for 3 months if $\mathrm{MO}$ is mild and in opinion of clinician likely to spontaneously improve (30\% chance).

(c) If mild to moderate macular ischaemia is present consider treatment with ranibizumab or Ozurdex if spontaneous improvement is unlikely.

(d) If severe macular ischaemia is present-no treatment is recommended, and regularly observe for NV formation.

3. If $\mathrm{VA} 6 / 12$ or worse, or there is progressive deterioration of $\mathrm{VA}+$ macular oedema and haemorrhages are masking macula.

(a) Monthly ranibizumab or baseline Ozurdex for 3 months.

(b) Perform FFA at 3 months to assess foveal integrity. (c) If severe macular ischaemia is found to be present at 3 months, then no treatment will likely be beneficial and further therapy should be carefully considered.

\section{At 3 months follow-up}

1. Consider modified-grid laser photocoagulation if persistent $\mathrm{MO}$, no or minimal macular ischaemia and other treatments unsuccessful or unavailable.

2. If $\mathrm{VA} \geq 6 / 9$ or no macular oedema detected, then continue to observe if initially observed. If on antiVEGF or Ozurdex therapy, then continue as suggested in $\mathrm{MO}$ due to CRVO.

\section{Further follow-up}

1. If under observation only, then follow-up 3 monthly intervals for 18 months.

2. In case of recurrence or new $\mathrm{MO}$, consider reinitiating intravitreal ranibizumab, or Ozurdex therapy.

\section{Ischaemic BRVO}

(a) Watch carefully for NV.

(b) If NVE-consider sector laser photocoagulation applied to all ischaemic quadrants. Intravitreal bevacizumab (off-licence) may also be given in combination with laser.

(c) Follow-up at 3 monthly intervals for up to 24 months.

\section{Clinical research in $\mathrm{RVO}$}

There are yet unreported studies comparing the efficacy of Ozurdex and anti-VEGFs in the treatment of MO secondary to RVO. The SCORE 2 study is comparing the visual outcomes of monthly bevacizumab and aflibercept as first line treatment for MO due to CRVO for 6 months, and then the trial will continue to evaluate the role of Ozurdex as a second line option. Similarly, there is an on-going NHIR HTA-CEAT funded study evaluating the efficacy of the different available anti-VEGFs in MO secondary to CRVO (LEAVO trial).

The surgical treatments for RVO currently remain experimental. Innovative therapies in the treatment of ischaemic RVOs, where current therapies are ineffective, may allow visual preservation or restoration.

\section{Conflict of interest}

SS has participated in Advisory Boards, and received educational travel grants and speaker fees from Bayer, Novartis, Allergan, and Roche. Her institution has received 
research grant funding from Allergan, Bayer, Novartis, and Roche. WMA has provided consultancy services to Alcon, Alimera, Allergan, Bayer, Novartis, and Thrombogenics. He has received travel grants from Alimera, Allergan, Bayer, and Novartis, and honoraria for lectures from Allergan and Novartis. He has participated in clinical trials for which his institution has received funding from Allergan, Novartis, and Pfizer. His institution has further received research grants from Allergan and Novartis for nonclinical studies, and CentreVue (Italy) for clinical studies. PH has participated in Advisory Boards, and received educational travel grants from Bayer, Novartis and Allergan. His institution has received research grant funding from Allergan, Bayer, and Novartis.

\section{Contributors}

\section{Members of the RVO Guideline Group}

Miss Sobha Sivaprasad, Moorfields Eye Hospital and Kings College Hospital, London, UK

Mr Winfried Amoaku, Nottingham University Hospital, Nottingham, UK

Mr Tom Williamson, Guy's and St Thomas Hospital, London, UK

Prof Paul Dodson, Birmingham Heartlands Hospital, Birmingham, UK

Mr James Talks, Royal Victoria Infirmary, Newcastle upon Tyne, UK

Dr Katherine Talks, Royal Victoria Infirmary, Newcastle upon Tyne, UK

Miss Khanchan Bhan, Moorfields Eye Hospital, London, UK

Mr Philip Hykin, Moorfields Eye Hospital, London, UK

\section{References}

1 Green WR, Chan CC, Hutchins GM, Terry JM. Central retinal vein occlusions: A prospective histopathologic study of 29 eyes in 28 cases. Retina 1981; 1: 27-55.

2 Green WR. Retina. In: Spencer WH (ed). Ophthalmic pathology. An Atlas and Textbook. 3rd ed. WB Saunders: Philadephia, 1985; p589.

3 Hockley DJ, Tripathi RC, Ashton N. Experimental retinal branch vein occlusion in the monkey. Histopathological and ultrastructural studies. Trans Ophthalmol Soc UK 1976; 96(2): 202-209.

4 Orth DH, Patz A. Retinal branch vein occlusion. Surv Ophthalmol 1978; 22: 357-376.

5 Natural history and clinical management of central retinal vein occlusion. The Central Vein Occlusion Study Group. Arch Ophthalmol 1997; 115: 486-491.

6 A randomized clinical trial of early panretinal photocoagulation for ischemic central vein occlusion. The Central Vein Occlusion Study Group N report. Ophthalmology 1995; 102: 1434-1444.

7 Sabates R, Hirose T, McMeel JW. Electrophysiology in the prognosis and classification of central retinal vein occlusion. Arch Ophthalmol 1983; 101: 232-235.
8 Hayreh SS, Klugman MR, Podhajsky P, Kolder HE. Electroretinography in central retinal vein occlusion. Correlation of electro-retinographic changes with pupillary abnormalities. Graefes Arch Clin Exp Ophthalmol 1989; 227: 549-561.

9 Breton ME, Quinn GE, Keene SS, Dahmen JC, Brucker AJ. Electroretinogram parameters at presentation as predictors of rubeosis in CRVO patients. Ophthalmology 1989; 96: 1343-1352.

10 Kay SB, Harding SP. Early electroretinography in unilateral central retinal vein occlusion as a predictor of rubeosis iridis. Arch Ophthalmol 1988; 106: 353-356.

11 Bresnick GH. Following up patients with central retinal vein occlusion. Arch Ophthalmol 1988; 106: 324-326.

12 Hayreh SS, Rojas P, Podhajsky P, Montague P, Woolson RF. Ocular neovascularisation with retinal vascular occlusion. III. Incidence of ocular neovascularisation with retinal vein occlusion. Ophthalmology 1983; 90: 488-506.

13 Quinlan PM, Elman MJ, Kaur Bhatt A, Mardesich P, Enger C. The natural course of central retinal vein occlusion. Am J Ophthalmol 1990; 110: 118-123.

14 Miturn J, Brown GC. Progression of nonischemic central retinal vein obstruction to the ischemic variant. Ophthalmology 1986; 93: 1158-1162.

15 Argon laser scatter photocoagulation for prevention of neovascularization and vitreous hemorrhage in branch vein occlusion. A randomized clinical trial. Branch Vein Occlusion Study Group. Arch Ophthalmol 1986; 104: 34-41.

16 Rogers S, McIntosh RL, Cheung N, Lim L, Wang JJ, Mitchell $\mathrm{P}$ et al. The prevalence of retinal vein occlusion: Pooled data from population studies from the United States, Europe, Asia, and Australia. Ophthalmology 2010; 117: 313-319.

17 Tait C, Baglin T, Watson H, Laffan M, Makris M, Perry D, Keeling D, British Committee for Standards in Haematology. Guidelines on the investigation and management of venous thrombosis at unusual sites. Br J Haematol 2012; 159(1): 28-38.

18 Evaluation of grid pattern photocoagulation for macular edema in central vein occlusion. The Central Vein Occlusion Study Group M report. Ophthalmology 1995; 102: 1425-1433.

19 Argon laser photocoagulation for macular edema in branch vein occlusion. The Branch Vein Occlusion Study Group. Am J Ophthalmol 1984; 98(3): 271-282.

20 Haller JA, Bandello F, Belfort Jr R, Blumenkranz MS, Gillies $\mathrm{M}$, Heier J et al. Dexamethasone intravitreal implant in patients with macular edema related to branch or central retinal vein occlusion twelve-month study results. Ophthalmology 2011; 118(12): 2453-2460.

21 Campochiaro PA, Brown DM, Awh CC, Lee SY, Gray S, Saroj $\mathrm{N}$ et al. Sustained benefits from ranibizumab for macular edema following central retinal vein occlusion: twelve-month outcomes of a phase III study. Ophthalmology 2011; 118(10): 2041-2049.

22 Brown DM, Campochiaro PA, Bhisitkul RB, Ho AC, Gray S, Saroj N et al. Sustained benefits from ranibizumab for macular edema following branch retinal vein occlusion: 12-month outcomes of a phase III study. Ophthalmology 2011; 118(8): 1594-1602.

23 Heier JS, Campochiaro PA, Yau L, Li Z, Saroj N, Rubio RG et al. Ranibizumab for macular oedema due to retinal vein occlusions: long-term follow-up in the HORIZON trial. Ophthalmology 2012; 119(4): 802-809. 
24 Brown DM, Heier JS, Clark WL, Boyer DS, Vitti R, Berliner AJ et al. Intravitreal aflibercept injection for macular oedema secondary to central retinal vein occlusion: 1-year results from the phase 3 COPERNICUS Study. Am J Ophthalmol 2013; 155(3): 429-437.

25 Korobelnik JF, Holz FG, Roider J, Ogura Y, Simader C, Schmidt-Erfurth $\mathrm{U}$, Lorenz $\mathrm{K}$ et al. Intravitreal aflibercept injection for macular edema resulting from central retinal vein occlusion. One-year results of the Phase 3 GALILEO Study. Ophthalmology 2014; 121: 202-208.

26 Campochiaro PA, Clark WL, Boyer DS, Heier JS, Brown DM, Vitti $\mathrm{R}$ et al. Intravitreal aflibercept for macular edema following branch retinal vein occlusion: the 24-week results of the VIBRANT study. Ophthalmology 2015; 122(3): $538-544$.
S Sivaprasad' ${ }^{1}$ WM Amoaku², P Hykin ${ }^{1}$ and RVO Guideline Group ${ }^{3}$

'Moorfields Eye Hospital, London, UK

${ }^{2}$ Queen's Medical Centre, Nottingham, UK

E-mail: senswathi@aol.com

${ }^{3}$ Members of the RVO Guideline Group are listed before the references

Link to full clinical guideline: https://www.rcophth.ac. uk/standards-publications-research/clinical-guidelines

Eye (2015) 29, 1633-1638; doi:10.1038/eye.2015.164; published online 28 August 2015 